\title{
Single-step fabrication of microfluidic channels filled with nanofibrous membrane using femtosecond laser irradiation
}

\author{
Amirhossein Tavangar ${ }^{1}$, Bo $\operatorname{Tan}^{2}$ and K Venkatakrishnan ${ }^{1,3}$ \\ ${ }^{1}$ Department of Mechanical and Industrial Engineering, Ryerson University, 350 Victoria Street, \\ Toronto, Ontario, M5B 2K3, Canada \\ ${ }^{2}$ Department of Aerospace Engineering, Ryerson University, 350 Victoria Street, Toronto, Ontario, \\ M5B 2K3, Canada \\ E-mail: venkat@ryerson.ca
}

Received 15 March 2010, in final form 4 June 2010

Published DD MMM 2010

Online at stacks.iop.org/JMM/20/000000

\begin{abstract}
In this research, we demonstrate a new method to fabricate silicon microfluidic channels filled with a porous nanofibrous structure utilizing a femtosecond laser. The nanofibrous structure can act as a membrane used for microfiltration. This method allows us to generate both the microfluidic channel and the fibrous nanostructure in a single step under ambient conditions. Due to laser irradiation, a large number of nanoparticles ablate from the channel surface, and then aggregate and grow into porous nanofibrous structures and fill the channels. Energy dispersive X-ray spectroscopy (EDS) analysis was conducted to examine the oxygen concentration in the membrane structure. Our results demonstrated that by controlling the laser parameters including pulse repetition, pulse width and scanning speed, different microfluidic channels with a variety of porosity could be obtained.

Q1 (Some figures in this article are in colour only in the electronic version)
\end{abstract}

\section{Introduction}

The usage of membranes in microfluidics has been growing rapidly [1]. Various recent studies have concentrated on the evolvement of membranes with nanometer-scale pores [2-5]. Several methods have been developed to fabricate nanoporous membranes such as lithography [6-8], ion track technique [9], self-organization [10] and sol-gel [11]. Most of these methods suffer from manufacturing complexity either in lithography processes or during membrane assembly in the microfluidic systems [2]. Despite its easy control of pore diameter and simple fabrication procedure, the ion track technique has limitations owing to both the inefficiency caused by the thick membrane and the inconvenient deployment in other microfluidic systems. In the sol-gel technique, the

\footnotetext{
3 Author to whom any correspondence should be addressed.
}

material is too costly and a ceramic support layer, fabricated by solid state sintering, is required [13].

Due to high chemical and thermal stability, silicon is an appealing substrate for microfluidic devices. Previous researches suggest that fabricated silicon membranes are inert and relatively nontoxic [14]. In addition, good mechanical strength of silicon membranes facilitates their utilization in pressurized filtering devices at macro- and micro-scale as well as in harsh environments [7].

Ultrafast laser micromachining of materials has been extensively employed over the last few decades, and its potential applications have been tried in numerous areas, particularly for analytic sampling, thin film deposition, surface micromachining, MEMS and NEMS fabrication, laser nanofabrication and surgical applications [15-20].

In this paper, we report a new laser application for fabrication of nanoporous membranes inside microfluidic channels which provides high controllability on average 


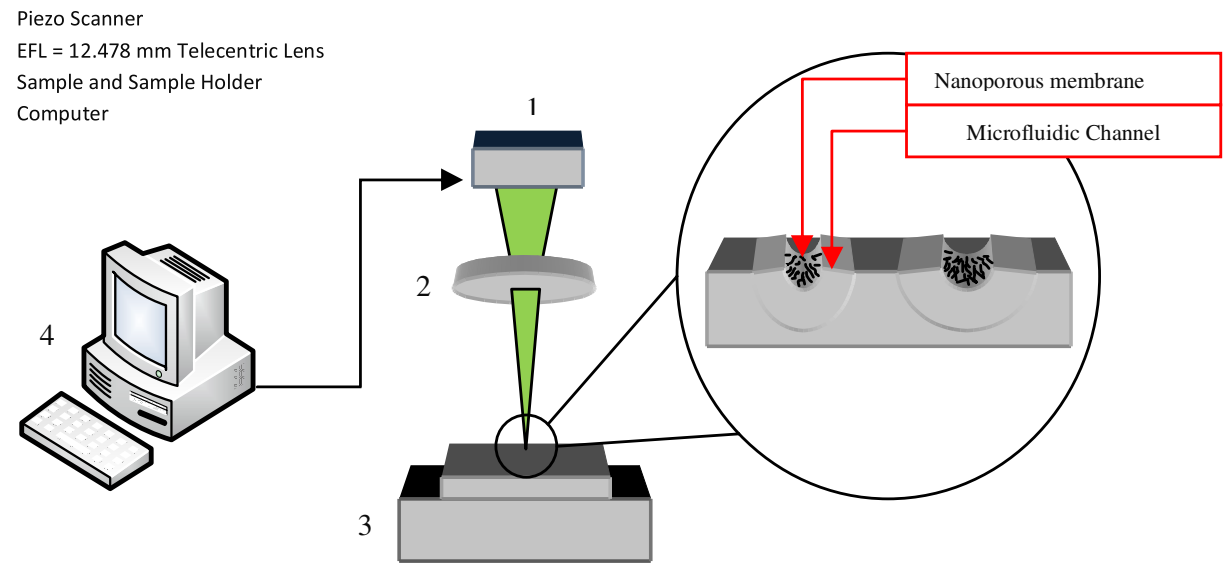

Figure 1. Experimental setup.

pore sizes. It is interesting to note that our proposed method generates both a porous nanofibrous structure and a microfluidic channel in a single step under ambient conditions. The femtosecond laser pulses hit a raw material and generate a microchannel along with a mass quantity of nanoparticles. These nanoparticles agglomerate to each other in a semi-solid state and form a nanofibrous structure in the channel. The nanoparticles are fused together rather than loosely bonded. Thus we assume that the bond structure of aggregation and the channel is strong. We have also determined that the pore size and density in the membrane can be controlled through adjustment of the laser parameters and a laser scanning procedure.

\section{Experimental setup and fabrication process}

Experiments were performed with a $1040 \mathrm{~nm}$ wavelength direct-diode-pumped $\mathrm{Yb}$-doped fiber-amplified ultrafast laser system. The maximum output power of the laser is $15 \mathrm{~W}$ and pulse repetition range is from $200 \mathrm{kHz}$ to $26 \mathrm{MHz}$. Because of solid-state operation and high spatial mode quality of fiber lasers, the Yb-doped fiber oscillator/fiber amplifier functions under low noise performance. In addition, parameters of the laser, such as laser repetition rate, pulse width and beam power, are computer monitored, which enables a simple interaction with the performed experiments. The target was a polished silicon wafer with $\langle 100\rangle$ crystal orientation and $550 \mu \mathrm{m}$ thickness. The schematic diagram of the experimental setup is shown in figure 1. The experimental setup has been described in detail in [21]. The specimens were then characterized using scanning electronic microscopy (SEM) followed by energy dispersive X-ray spectroscopy (EDS) analyses.

\section{Results and discussions}

\subsection{The growth mechanism of a nanofibrous structure}

Primary experiments were conducted to investigate laser scanning strategies for the creation of a microchannel filled with a nanofibrous structure including laser scanning speed and number of passes. The close scheme of the nanofiber growth mechanism in a microchannel and its cross-sectional views are shown in figure 2.

Basically, focusing a laser beam at a target creates a heated region which causes vaporization leading to formation of plasma that consists of electrons and ionized atoms. As the plasma expands outward, due to heat transfer at the contact surface between the plume and the ambient gas, the vapor plume starts to cool down and condensation begins at the cooler front of the plume cloud [22, 23]. Condensation of the vaporized atoms leads to nucleation and consequently the growth of nanoparticles [24]. Nanoparticle aggregation takes place as a part of vapor condensation by the collision of nuclei. An immense amount of nanoparticle aggregation is required in order to generate nanofibrous structures. Therefore, continuous arrival of the plume is needed before the pulse cools below the vaporization point. A close view of the nanofibrous structures exhibits that the nanoparticles are mostly fused together instead of being loosely linked. The structures are made of self-assembled closed-ring and bridge nanofibers. Therefore, the bond between the aggregated structure and the channels is assumed to be strong.

As mentioned above, the plumes generated by sequential laser pulses have to arrive at the target before the critical time. Hence, the formation of a fibrous structure is not possible in high speed laser machining. Therefore, as the first step, optimum laser machining speed must be defined. As the machining speed decreases, the interaction time between the laser beam and the target increases, leading to a higher laser pulse per spot on the target which consequently results in a higher amount of nanofiber agglomeration. On the other hand, by controlling laser scanning passes, a variety of microchannels with different depths and widths can be achieved.

\subsection{Effect of laser scanning parameters}

As depicted in figure 3, it was found that at low laser scanning speeds and high scanning passes, the depth of the microfluidic channel increases and its width decreases. In the case of high number of laser scanning passes, a more fibrous structure is generated. 


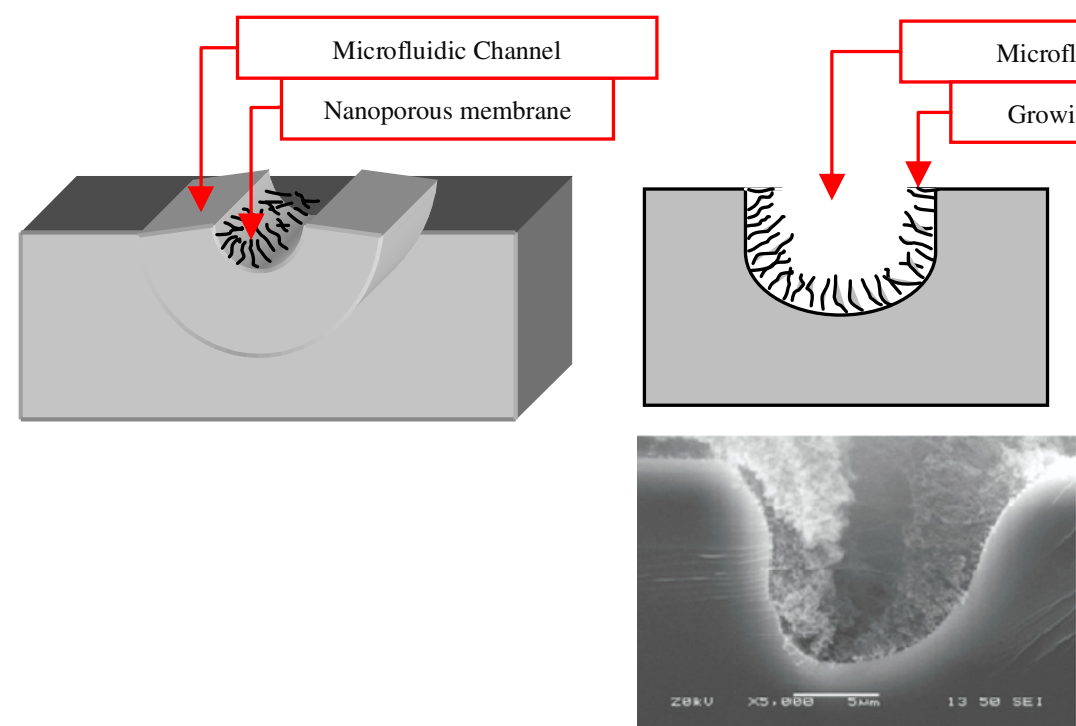

(a)

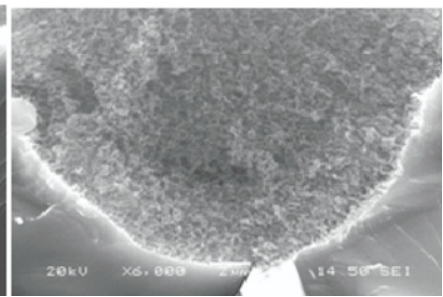

(b)

Figure 2. 3D and plane view of the microchannel and sequences of nanofiber growth inside the microchannel. The related SEM images corresponding to each sequence are shown at the bottom. The microchannels were formed by a laser beam at the frequency of $13 \mathrm{MHz}$ with the laser scanning speed of $1000 \mathrm{~mm} \mathrm{~s}^{-1}$ after $(a) 5$ and $(b) 20$ scanning passes.

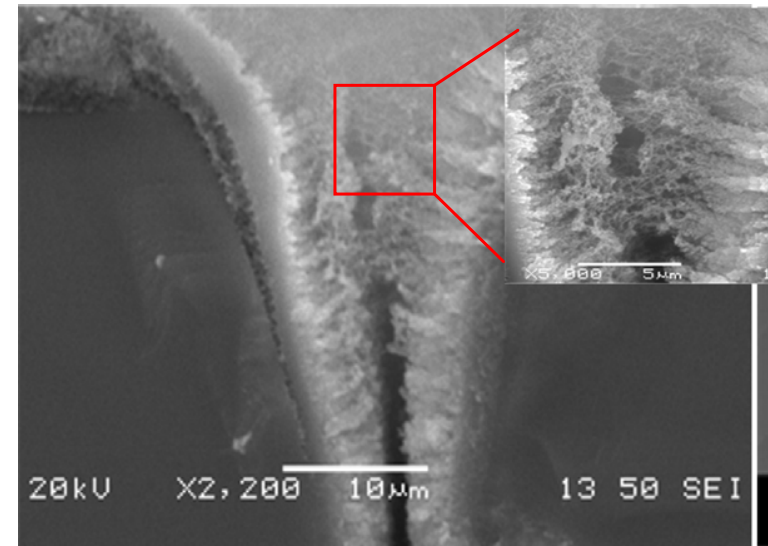

(a)

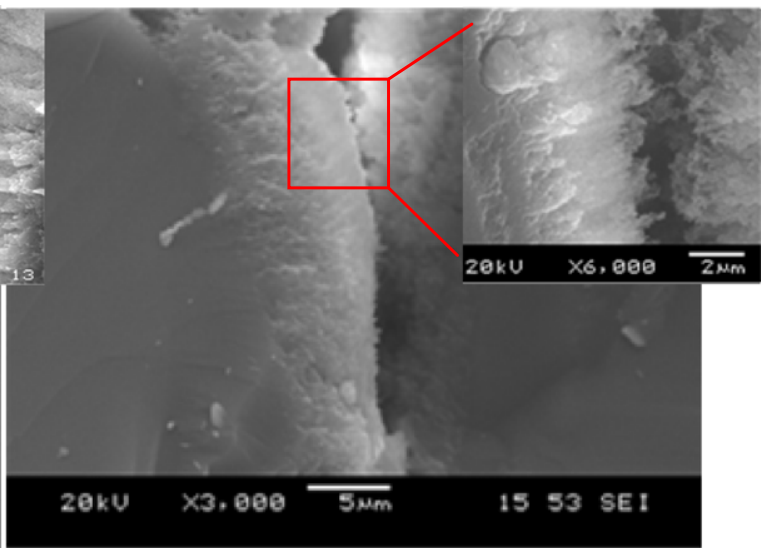

(b)

Figure 3. SEM cross-sectional image of the nanofibrous structure inside the microfluidic channel formed by the laser beam at the frequency of $13 \mathrm{MHz}$ with the laser scanning speed of $(a) 1000 \mathrm{~mm} \mathrm{~s}^{-1}$ and (b) $2000 \mathrm{~mm} \mathrm{~s}^{-1}$.

In addition to its effect on the number of generated nanofibrous structure, laser scanning speed influences the microchannel shape as well. Comparing figures 3(a) and (b), at lower scanning speed (figure 3(a)), a narrower channel was formed because of more dwell time on a specific spot and consequently higher energy per pulse. Conversely, at higher speed (figure 3(b)), the plumes generated by sequential laser pulses could not arrive at a particular spot before the critical time needed to maintain the nucleus density at a certain level; therefore, no nanofibers were generated.

Figure 4 shows the growth mechanism of the nanofibrous structure for different laser scanning passes. Firstly, microchannels were generated at high scanning speed with few scanning passes. As mentioned before, at higher speed, due to low interaction time between the laser and the substrate, no fibrous structure was generated, and only the microchannel was formed. Afterward, low scanning speed was employed to grow nanofibers inside the channel. As the number of scanning passes increases, more fibrous structures grow on the microchannel walls.

\subsection{Study of pore size and density of the nanofibrous structure}

SEM analyses of the nanofibrous structure revealed that laser repetition rate and laser pulse width have an important impact on the pore size and density of the fibrous structure.

3.3.1. Effects of laser repetition rate. After finding the optimum laser scanning strategy, the effects of the laser repetition rate on both the shape of the channels and the porosity of the nanofibrous structure were determined.

In the case of multiple-pulse irradiation, a massive nanoparticle agglomeration takes place if the succeeding pulse arrives before the pulse cools below the vaporization point. 


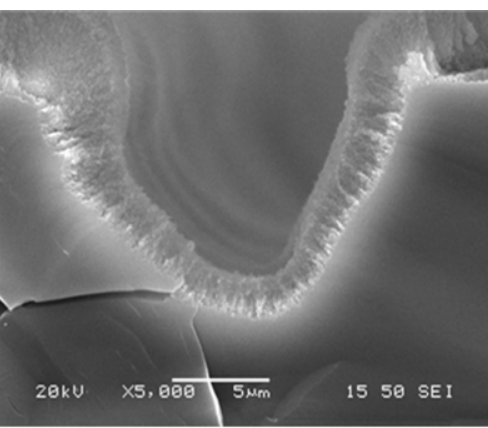

(a)

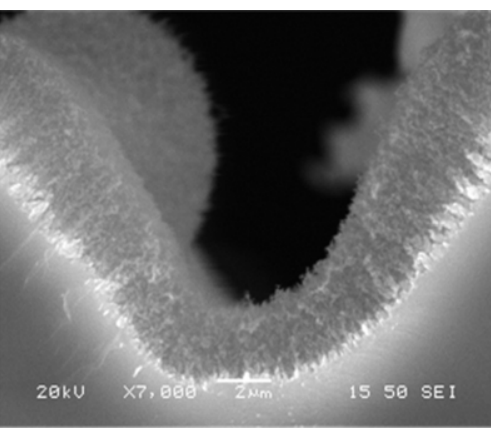

(b)

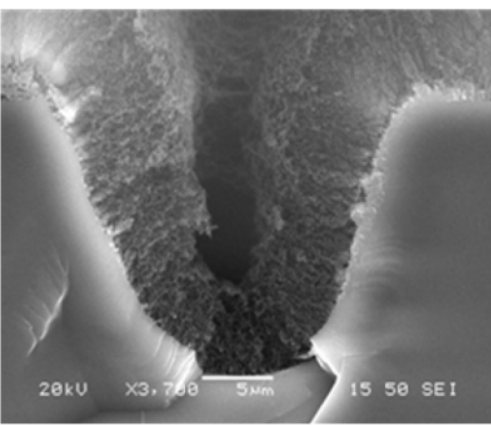

(c)

Figure 4. SEM cross-sectional image of nanofibrous structure growth sequences inside the microfluidic channel formed by a laser beam of $1040 \mathrm{~nm}$ wavelength at the frequency of $26 \mathrm{MHz}$ after (a) 5, (b) 10 and (c) 20 laser scanning passes.
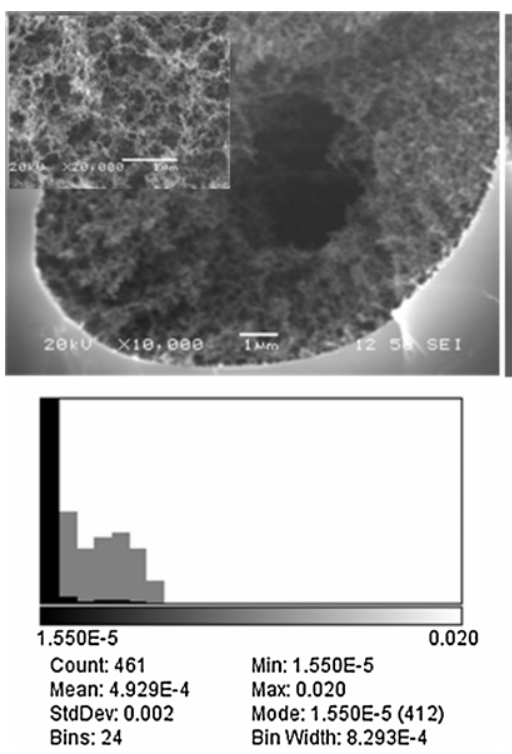

(a)
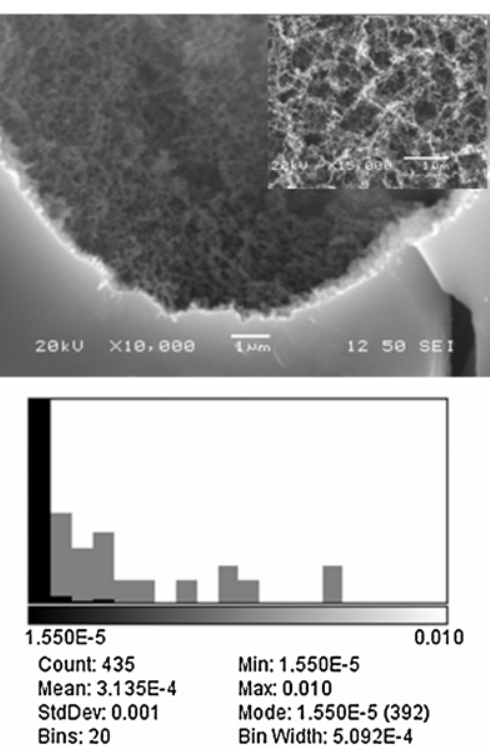

(b)
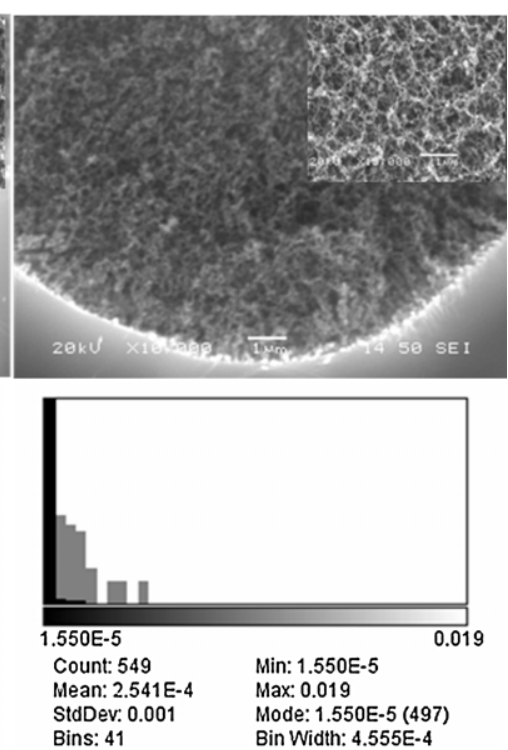

(c)

Figure 5. SEM cross-sectional image and the pore diameter distribution graph of the nanofibrous structure inside the microfluidic channel formed by the laser beam of $1040 \mathrm{~nm}$ wavelength, at the frequency of (a) $8 \mathrm{MHz}(b) 13 \mathrm{MHz}$ and (c) $26 \mathrm{MHz}$.

Therefore, the experiments were conducted at the frequencies of 8,13 and $26 \mathrm{MHz}$ to obtain the most nanofibrous structure. Note that in our experiments, the time interval between pulses is about $125 \mathrm{~ns}, 77 \mathrm{~ns}$ and $38 \mathrm{~ns}$ for the frequencies of 8 , 13 and $26 \mathrm{MHz}$, respectively. One of the distinguishing characteristics of these nanofibrous structures, unlike the random heaping of particles observed at $\mathrm{Hz}$ and $\mathrm{kHz}$ pulse frequencies, is that the fiber aggregation shows a certain degree of organization.

Pore size data were extracted directly from the SEM images using the image processing software. The SEM images of these membranes (see figure 5) revealed that the membrane pore size decreases as the pulse repetition rate increases, whereas the density of pores increases with increasing pulse repetition rate. However, pore size distribution is wider for $13 \mathrm{MHZ}$ repetition in comparison to the other two frequencies. This heterogeneity might have been caused when the channels got cut for cross-sectional imaging. Membranes have an average pore diameter of $492 \mathrm{~nm}, 313 \mathrm{~nm}$ and $254 \mathrm{~nm}$ at the frequencies of 8,13 and $26 \mathrm{MHz}$, respectively. The minimum pore diameter has been calculated to be approximately $90 \mathrm{~nm}$ at the repetition of $26 \mathrm{MHz}$.
At the pulse repetition rates higher than the nanoparticle formation threshold, consecutive laser pulses hit the substrate before the ablated particles from the previous pulse cool down. As a result, nanoparticles ejected by the following laser pulses prefer to aggregate with particles ablated from the former laser pulses which are still at a very high temperature, resulting in an increase in nanofibrous structure density. Moreover, since laser power and laser spot size are constant, the pulse energy decreases as pulse repetition increases which results in a reduction in the nanoparticle size. Consequently, because of the smaller nanoparticle size, nanofibers become finer, leading to the lessening of the pore size (see figure 5).

3.3.2. Effects of pulse width. Afterward, the influence of the laser pulse width on the membrane pore size was investigated. The laser used for all the presented experiments has the ability to work with different pulse widths from 214 fs to 3.5 ps.

To investigate the influence of different laser pulse widths on the porosity of the membrane, experiments were conducted at the repetition of $26 \mathrm{MHz}$ with the same laser scanning speed and number of passes, but with 

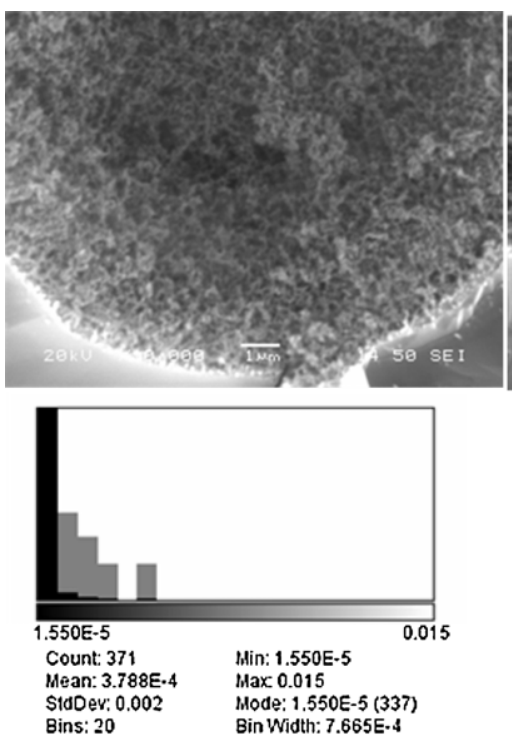

(a)
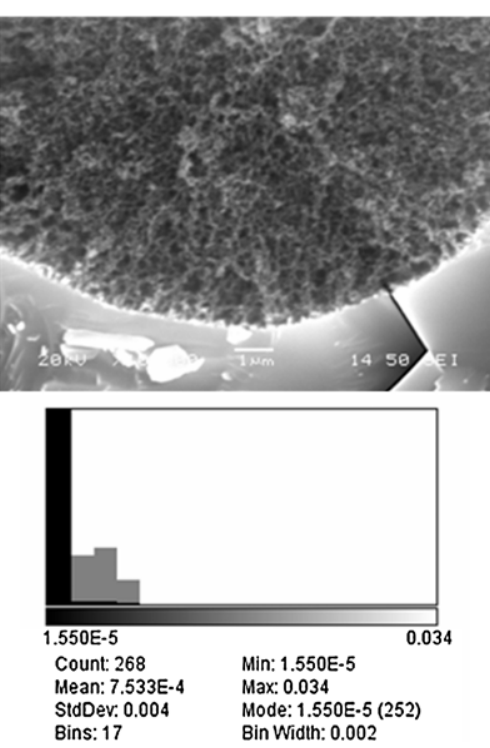

(b)
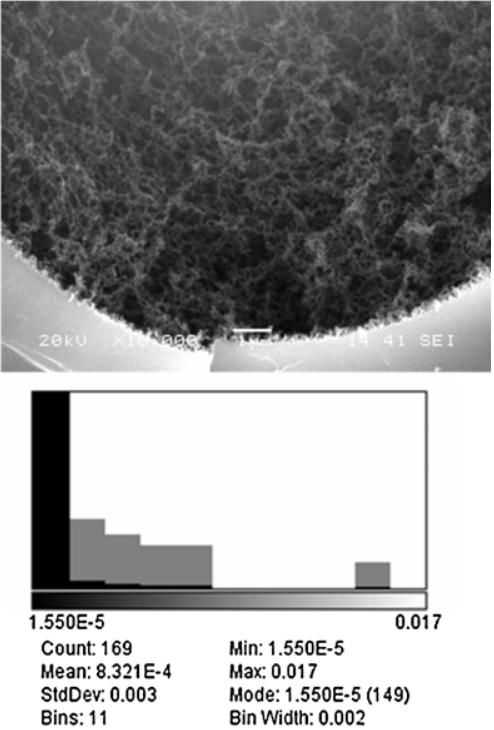

(c)

Figure 6. SEM image and the pore diameter distribution graph of the nanofibrous structure inside the microfluidic channel formed by a laser beam at the frequency of $26 \mathrm{MHz}$ with pulse width of $(a) 214 \mathrm{fs}(b) 1428 \mathrm{fs}$ and $(c) 3500 \mathrm{fs}$.
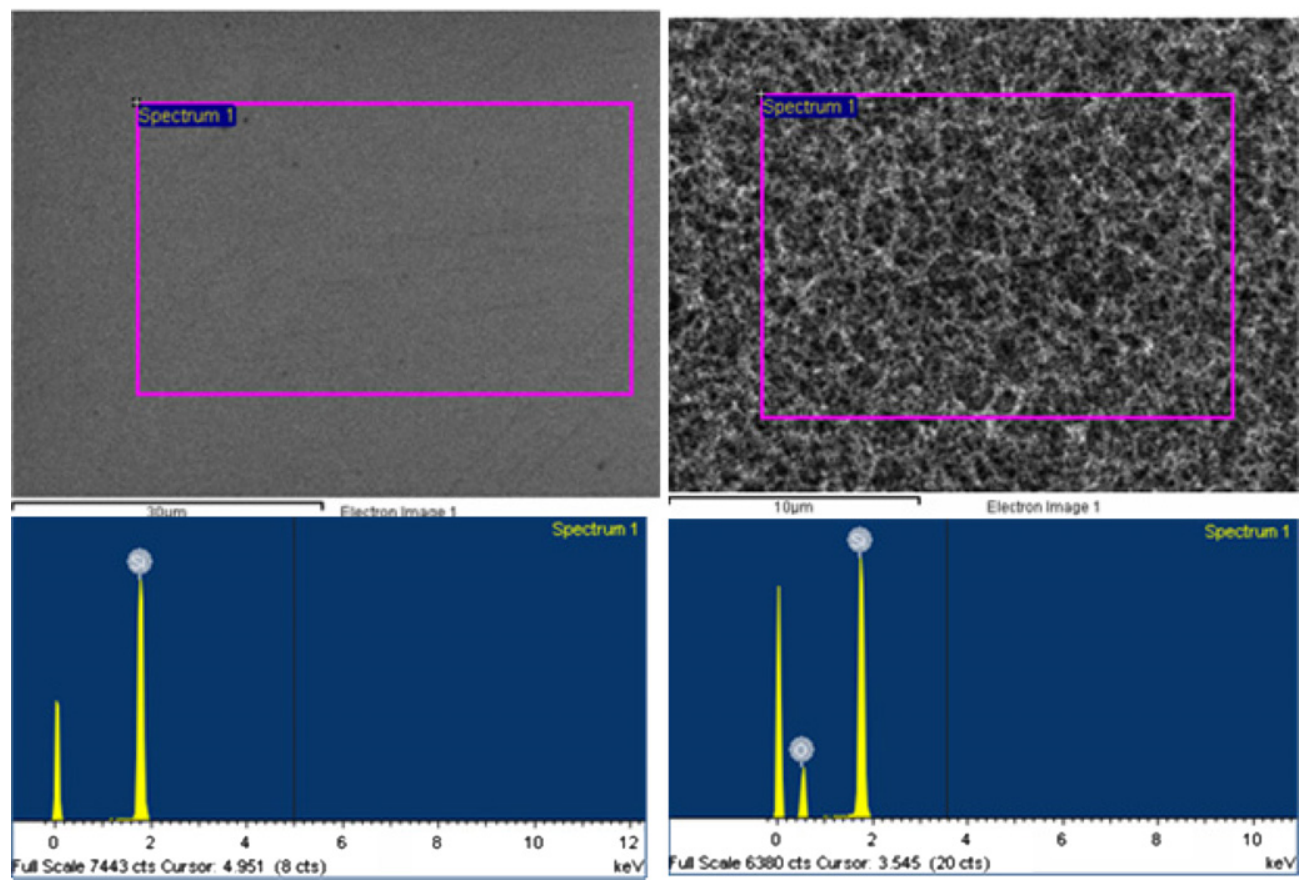

Figure 7. Energy dispersive x-ray spectroscopy (EDS) analysis of pure Si (left) and silicon oxide nanofibers deposited on a Si substrate (right).

different pulse widths of 214, 1428 and 3500 fs (see figure 6). The experiments showed that as the pulse width goes to the femtosecond scale, less nanofibrous structures are generated. As the pulse width decreases from picoseconds to femtoseconds, the interaction time between the laser pulse and the substrate decreases. This causes a decrease in the size of the nucleus which forms the nanoparticles. Accordingly, the size of nanofibers which are formed by nanoparticle agglomeration decreases. As a result, the smaller nanofibers lead to pore size reduction (see figure 6). The average pore diameters of the membranes were about $378 \mathrm{~nm}, 753 \mathrm{~nm}$ and $832 \mathrm{~nm}$ at pulse widths of $214 \mathrm{fs}, 1428 \mathrm{fs}$ and $3500 \mathrm{fs}$, respectively. The smallest pore diameter has been estimated to be around $160 \mathrm{~nm}$ at $3500 \mathrm{fs}$ pulse width.

Finally, in order to examine the composition of nanofibrous structures, EDS analysis, an integrated feature of a scanning electron microscope, was performed. Figure 7 depicts the EDS analysis comparing generated nanofibrous structures with an un-doped Si wafer. Since experiments were carried out under ambient conditions, the presence of oxygen along with silicon $(\mathrm{Si})$ is noticeable for the nanofibrous structures. 


\section{Conclusion}

We have developed a new method for fabricating silicon dioxide nanofibrous membranes grown inside microchannels using the femtosecond laser. The experiments showed that by controlling the laser parameters including pulse repetition, pulse width and scanning speed, different microfluidic channels with different porosities and pore densities could be obtained. The EDS analysis indicated oxygen concentration in the membrane structure which was attributed to oxidation of ablated material in ambient atmosphere. Using this strategy, the microchannel and the fibrous nanostructure for a wide variety of materials can be generated in a single step under ambient conditions. This achievement can, in the future, pave the way for extensive application of nanofilters in various membrane/filtration/separation applications.

\section{Q3 References}

[1] Frenot A and Chronakis I S 2003 Curr. Opin. Colloid. Interface Sci. 8 64-75

[2] Cooper S M, Cruden B A, Meyyappan M, Raju R and Roy S 2004 Nano Lett. 4 377-81

[3] Holt J K, Noy A, Huser T, Eaglesham D and Bakajin O 2004 Nano Lett. 4 2245-50

[4] Albu S P, Ghicov A, Macak J M, Hahn R and Schmuki P 2007 Nano Lett. 7 1286-9

[5] Mao P and Han J 2009 Lab Chip 9 586-91

[6] Tong H D, Jansen H V, Gadgil V J, Bostan C G, Berenschot E, van Rijn C J M and Elwenspoek M 2004 Nano Lett. 4 283-7

[7] Striemer C C, Gaborski T R, McGrath J L and Fauchet P M 2007 Nature 445 749-53
[8] Neeves K B and Diamond S L 2008 Lab Chip 8 701-9

[9] Zhang W M, Li J, Cao L X, Wang Y G, Guo W, Liu K X and Xue J M 2008 Nucl. Instrum. Methods Phys. Res. B $2663166-9$

[10] Yamaguchi A, Uejo F, Yoda T, Uchida T, Tanamura Y, Yamashita T and Teramae N 2004 Nat. Mater. 3 337-41

[11] Klein L C and Gallagher D 1988 J. Membr. Sci. 39 213-20

[12] Kim J and Gale B K 2008 Lab Chip 8 1516-23

[13] Lim G T, Jeong H G, Hwang I S, Kim D H, Park N and Cho J 2009 Desalination 238 53-9

[14] Kotzar G, Freas M, Abel P, Fleischman A, Roy S, Zorman C, Moran J M and Melzak J 2002 Biomaterials 23 2737-50

[15] Bogaerts A, Chen Z, Gijbels R and Vertes A 2003 Spectrochim. Acta B 58 1867-93

[16] Chrisey D B, Pique A, McGill R A, Horwitz J S, Ringeisen B R, Bubb D M and Wu P K 2003 Chem. Rev. 103 553-76

[17] Chen T C and Darling R B 2005 J. Mater. Process. Technol. $169214-8$

[18] Tavangar A, Tan B and Venkatakrishnan K 2010 J. Micromech. Microeng. 20055002

[19] Meijer J, Du K, Gillner A, Hoffmann D, Kovalenko V S, Masuzawa T, Ostendorf A, Poprawe R and Schul W 2002 CIRP Ann. 50 531-50

[20] Hosokawa C, Kudoh S N, Kiyohara A, Hosokawa Y, Okano K, Masuhara H and Taguchi T 2008 Appl. Phys. A 93 57-63

[21] Tan B, Dalili A and Venkatakrishnan K 2009 J. Appl. Phys. A 95 537-45

[22] Tillack M S, Blair D W and Harilal S S 2004 Nanotechnology 15 390-403

[23] Anisimov S I and Luk'yanchuk B S 2002 Phys._Usp. 45 293-32

[24] Lescoute E, Hallo L, Hébert D, Chimier B, Etchessahar B, Tikhonchuk V T, Chevalier J M and Combis P 2008 Phys. Plasmas 15 063507-18 


\section{QUERIES}

\section{Page 1}

Q1

Author: Please be aware that the colour figures in this article will only appear in colour in the Web version. If you require colour in the printed journal and have not previously arranged it, please contact the Production Editor now.

\section{Page 2}

Q2

Author: Please check whether the edits made in the sentence 'Basically, focusing a laser beam...' retain your intended sense.

\section{Page 6}

Q3

Author: Please check the details for any journal references that do not have a blue link as they may contain some incorrect information. Pale purple links are used for references to arXiv e-prints.

Q4

Author: Reference [12] is not cited in the text. Please check. 